\title{
La representación del "supervisor de las cosas selladas" Neshi en la Segunda Estela de Kamose (K2)
}

\section{The Representation of the "Overseer of Sealed Things" Neshi in the Second Stela of Kamose (K2)}

\author{
ROXANA FLAMMINI \\ Universidad Católica Argentina, Argentina
}

Resumen: La Segunda Estela de Kamose, un monumento conmemorativo regio erigido en el templo de Amón en Karnak, preserva la inusual imagen del "supervisor de las cosas selladas" Neshi en el ángulo inferior izquierdo. El funcionario fue el encargado de realizar la estela y colocarla en el templo por orden del rey. La figura se encuentra orientada hacia al texto escrito, y el funcionario sostiene en su mano izquierda un abanico ceremonial. En este trabajo propongo una explicación alternativa a la presencia de la figura del funcionario en la estela.

Palabras clave: Neshi; Segunda Estela de Kamose; Segundo Periodo Intermedio; Karnak; imagen-texto.

Recepción: 18 de abril de 2020. / Aceptación: 22 de junio de 2021.

D.R. ${ }^{\circ}$ 2022. Estudios de Asia y África Licencia Creative Commons Atribución-NoComercial-SinDerivar (CC BY-NC-ND) 4.0 Internacional 
Abstract: The unusual carved image of the "overseer of sealed things" Neshi is preserved in the lower left part of the Second Stela of Kamose, the commemorative royal monument erected in the temple of Amun at Karnak. The official was commissioned to make the stela and place it in the temple on orders from the king. The figure faces the written text, and the official holds a ceremonial fan in his left hand. In this paper, I propose an alternative explanation for the presence of the official's image in the stela.

Keywords: Neshi; Second Stela of Kamose; Second Intermediate Period; Karnak; image-text.

\section{Introducción}

La Segunda Estela de Kamose (Museo de Luxor, J. 43; PM II, 37; de aquí en adelante $\mathrm{K} 2$ ), una estela monumental regia de carácter conmemorativo, es conocida por ser una de las pocas narrativas que contiene información contemporánea sobre las guerras desatadas entre los tebanos, los asiáticos y los nubios a finales del Segundo Periodo Intermedio (mediados del segundo milenio a.e.c.). Por cierto, se debe tener en cuenta que en la narrativa predomina el sesgo particular de los tebanos en ese marco espaciotemporal. El texto recorre los enfrentamientos, da precisiones sobre varias localidades e incluye una carta que el gobernante hicso Apepi le envió al rey de Kush, y que fue interceptada por Kamose en el camino y devuelta al remitente. Al final del texto, se narra el regreso triunfal de $\mathrm{Ka}$ mose a Tebas, y, en el colofón, está la orden dirigida al "supervisor de las cosas selladas" Neshi, de realizar una estela que conmemorara las victorias del rey para que fuera instalada en el templo de Karnak (imagen 1). Su excelente estado de conservación, el conocimiento de su datación y de su lugar de destino hacen de este monumento uno de los más relevantes para el estudio de la fase final del Segundo Periodo Intermedio (Habachi 1972).

La estela posee decoración en dos sectores: en la luneta se visualiza un disco solar alado que sostiene dos uraei. Entre ellos 


\section{ImAgEN 1. Segunda Estela de Kamose, Museo de Luxor J43}

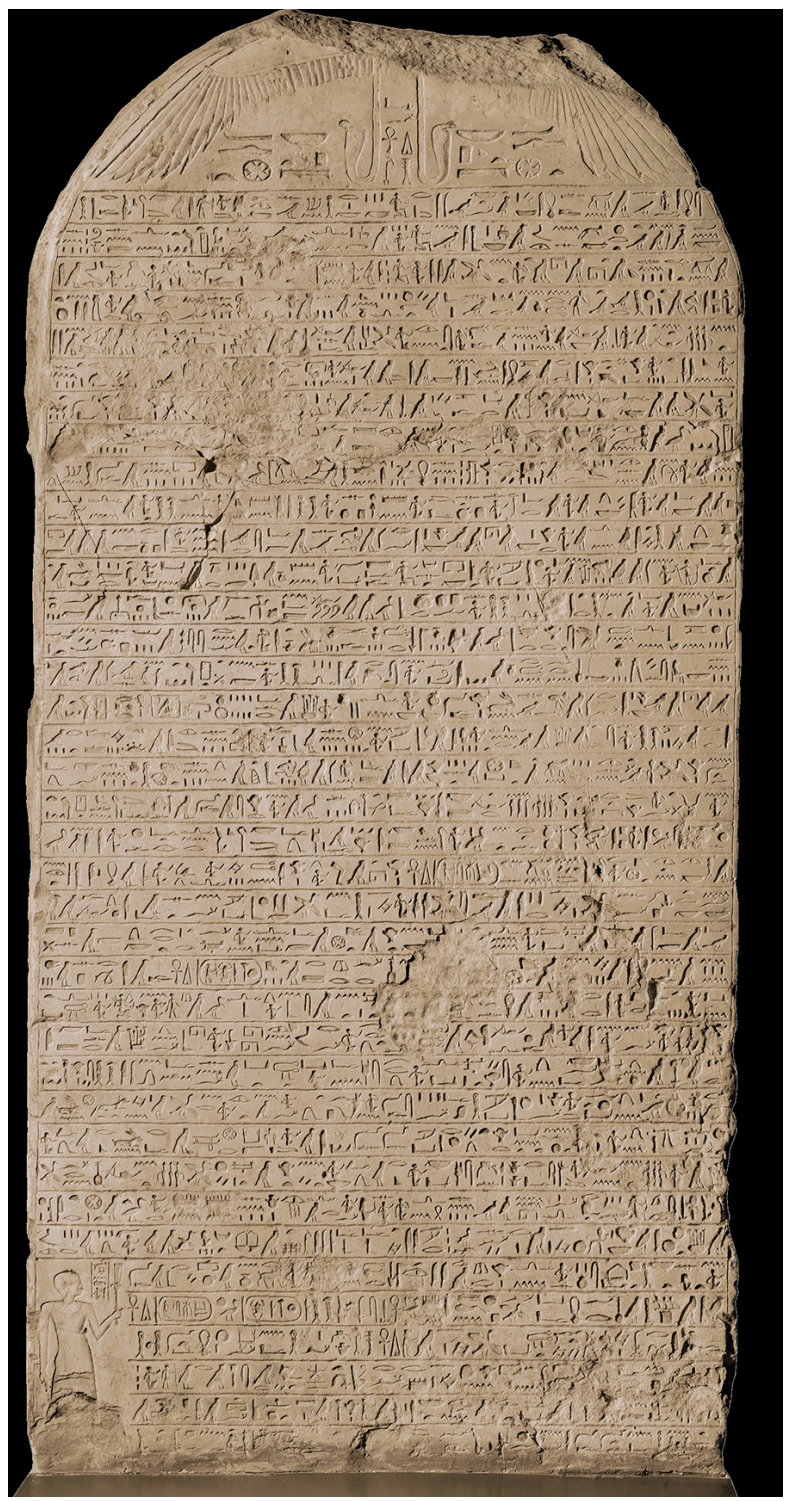

Fuente: Biston-Moulin y Thiers 2017, 62/J. Maucor. 
se encuentra una inscripción vertical que puede leerse como $r d j=f d j$ ' $n h \underline{d} \underline{d} d w 3 s$, "él (el dios) hace que le sea dada vida, estabilidad y poder a él (el rey)". Por debajo del disco alado hay una inscripción que se visualiza a ambos lados de los uraei y que puede leerse como Bhdt $n b$ pt, "el de Behedet, señor del cielo”, referencia que remite al Horus de Edfu. En el ángulo inferior izquierdo se encuentra la figura de Neshi, el funcionario a quien le fuera encargada la estela, orientada en dirección a lo narrado (imagen 2). Por cierto, esta representación no recibió la misma atención que otras particularidades del monumento, con lo cual aquí procuraré discernir su posible significación.

IMAgEN 2. Parte inferior de la Segunda Estela de Kamose, imagen de Neshi

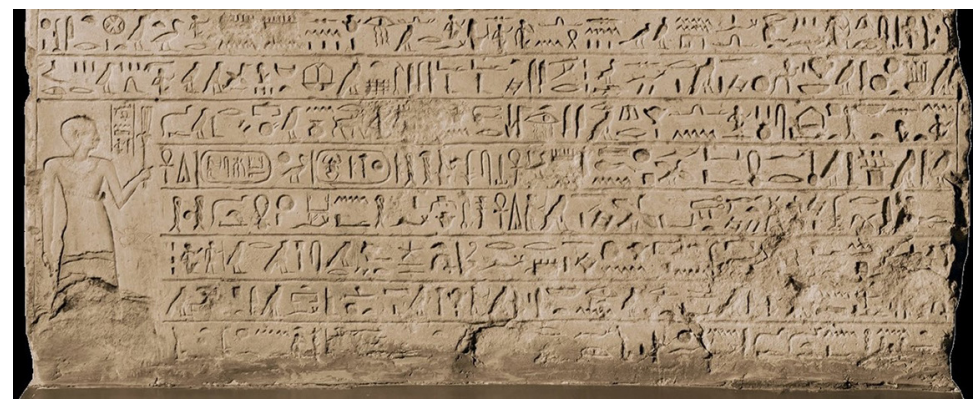

Fuente: Biston-Moulin y Thiers 2017, 62/J. Maucor.

La representación del "supervisor de las cosas selladas" Neshi en K2: interpretaciones previas, descripción y evidencias relacionadas

Pocos especialistas hicieron referencias explícitas al posible significado de la inusual presencia de la imagen de un funcionario en una estela de este tipo; de hecho, la atención estaba 
dirigida en mayor medida a identificar a Neshi. Así, en cuanto a la primera cuestión, G. Posener (1964, 213-214) consideró que se trató de un favor excepcional del rey a su funcionario, y, en cuanto a la segunda, propuso que el 'supervisor de los barcos' Neshi mencionado en la Inscripción de Mes (datada en el reinado de Ramsés II; cf. Gardiner 1905) -y que había recibido tierras en recompensa por sus acciones contra los hicsos por parte del sucesor de Kamose, el rey Ahmose (dinastía XVIII)- y el Neshi mencionado en K2 serían la misma persona. Otros autores siguieron esa propuesta, como L. Habachi $(1972,57)$ y, más recientemente, S. Allam $(1989,104$, n. 7). Habachi sostuvo, además, que era probable que Neshi tuviera un papel activo en las campañas navales de Kamose, al pertenecer a la armada real. Sin embargo, que ambos individuos hayan sido la misma persona es una hipótesis difícil de demostrar, ya que el cargo del Neshi de la Inscripción de Mes difícilmente puede ser equiparado con la posición encumbrada del Neshi representado en $\mathrm{K} 2$.

Ahora bien, a diferencia de estos especialistas, $\mathrm{H}$. Goedicke se refirió más a la cuestión de la inserción de la representación de Neshi en K2 que a su identificación, y propuso que el funcionario actuó como regente del rey mientras éste se encontraba fuera de Tebas debido a las guerras contra los hicsos, y era a quien el rey se reportaba cuando no estaba en la ciudad. Asimismo, sugirió que pudo haber utilizado su posición como regente para hacer un memorial a su persona en la propia estela (Goedicke 1971-1972, 152), luego de que Kamose perdiera la vida en la guerra contra los hicsos (Goedicke 1995, 99). En otras palabras, atribuyó a Neshi una función cuasi regia, capaz de habilitarlo para representarse a sí mismo en la estela. Y si bien esta última apreciación es sumamente especulativa, Goedicke también sostuvo que el papel ostentado por Neshi era de una gran cercanía al rey y ejercía una de las posiciones más altas en la estructura político-administrativa de la Tebas de la época. En este punto no podemos más que coincidir, aunque aquí propongo una interpretación alternativa para la inclusión de 
IMAGEN 3. Representación de Neshi, detalle

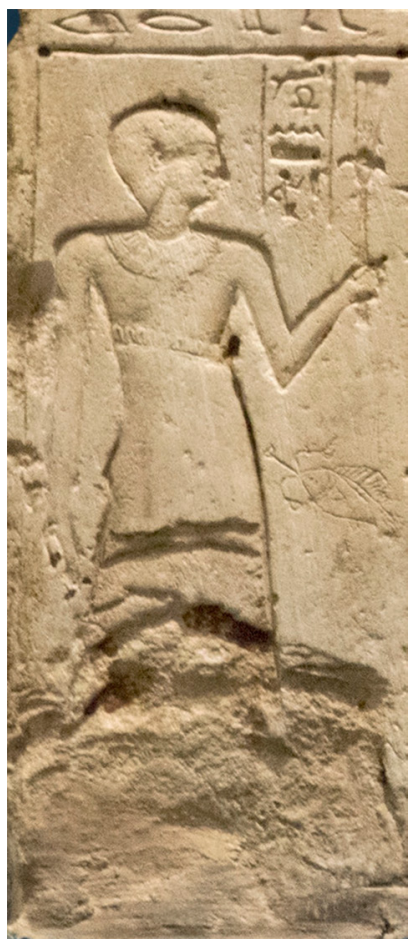

Fuente: Biston-Moulin y Thiers 2017, 62/J. Maucor.

su figura en $\mathrm{K} 2$, sin que necesariamente se descarten cuestiones ligadas al alto estatus del funcionario que le permitieran su propia representación.

Para ello consideraré, por un lado, el contexto (el Sitz im Leben) donde se inserta la representación de $\mathrm{Ne}$ shi, y, por otro, evidencias relativas a otros altos funcionarios de la Tebas del Segundo Periodo Intermedio que, si bien no son abundantes, resultan significativas.

La representación de $\mathrm{Ne}$ shi (imagen 3) está enmarcada por el regreso triunfal a Tebas de Kamose, quien se dirige al templo de Amón para realizar diversos rituales: quemar incienso en el "lugar santísimo" del templo y recibir el jepesh (espada con hoja en forma de hoz) de parte del dios como reconocimiento a su accionar militar. En este pasaje es llamado "hijo de Amón”, y es donde los nombres de coronación y de nacimiento del rey están escritos:

(31) "Me moví rápidamente hacia el distrito de Tebas en la estación de (32) la inundación. Cada rostro estaba brillante. [La] tierra en abundancia. La costa se volvió resplandeciente. Tebas en festival, mujeres [y] hombres salieron para ver(33)me, todas las mujeres abrazando a sus compañeros, ningún rostro en lágrimas. Yo quemé incienso para Amón en el Lugar Santísimo”. 
En el lugar donde es dicho: (34) “irecibe cosas buenas!”, él [i.e.: el dios] le dio el jepesh al hijo de Amón [que viva, esté próspero y sano], el rey viviente Wady-jeper-Ra, el hijo de Ra, Kamose el fuerte/valiente, a quien es dada vida (35), el que somete al sur y el que repele al norte, quien conquistó esta tierra victoriosamente, a quien es dada vida, estabilidad y poder. Su corazón [está] feliz junto con su ka, como Ra eternamente.

A continuación, el texto refiere, en primera persona del singular y en voz activa, lo dicho por el rey:

(36) Decreto de Su Majestad [dirigido al] miembro de la élite; líder de acción; guardián de los asuntos privados del palacio; líder de la tierra entera; portador del sello del rey [gobernante]; primer conductor/líder [?] de las Dos Tierras; supervisor de los amigos [del rey]; (37) supervisor de las cosas selladas, el poderoso Neshi:

"Pon todo lo que Mi Majestad ha hecho en victoria en una estela. Establece su lugar en Karnak, en (38) Tebas, por los siglos de los siglos".

\section{A lo que continúa la respuesta de Neshi:}

[Entonces] él [Neshi] dijo delante de su majestad: "Voy a actuar [de acuerdo con lo que me ha orden]ado [mi] señor, para que perdure mi favor junto al rey". 1

${ }^{1}$ La parte final de la línea 38 presenta dificultades para su reconstrucción debido al mal estado de conservación en el que se encuentra la piedra. Sin embargo, se efectuaron diversas propuestas a lo largo del tiempo. Así, Habachi (1972, 44, n. e) reconstruyó: irr.(i htf) $w(\underline{d}) t w . n .(n . i) n b(t) w \underline{d} h s t n t \underline{h r} n s w t$, "Actuaré de acuerdo con todo lo que es ordenado, y favores del rey serán otorgados" ("I shall act in accordance with all that is commanded and favors of the king were granted"); por su parte, H. Smith y A. Smith (1976, 66, n.gg), diferenciándose de Habachi, propusieron: irr.i hft wd it.i nb.i wh hh htst(i) $n t h r-n s w$, "Es de acuerdo con la orden de mi padre, mi señor, que yo actúo, para que mi favor pueda florecer delante del rey" ("It is in accordance with the command of my father, my lord that I act, in order that my favour may flourish before the King”). Por su parte, W. Helck (1983, 97), en su reconstrucción jeroglífica, presentó: $j r r=j[w p] w t n b[. t]$ h s.t $t=j n t h r-n s w t$, "Haré ejecutar cada trabajo para satis-

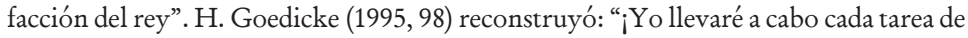
acuerdo con la alabanza de uno-con-el-Rey!" ("I shall carry out every commission according to the praise of one-with-the-king!”), mientras que D. Redford $(1997,15)$ tradujo: "Llevaré a cabo todo el encargo para satisfacción del rey" ("I will perform every assignment to the satisfaction of the king”). U. Kaplony-Heckel y E. Kausen (1985, 
A la derecha de la representación de Neshi, escrito en columna, se reitera el título de función y el nombre del funcionario: "El supervisor de las cosas selladas Neshi". Esta duplicación del nombre (en la narración y en la columna), y el hecho de que en la narración esté acompañado del término 粗 wsr, "poderoso", y que algunos de los signos estén dañados, abrió la posibilidad a interpretaciones diversas. La reconstrucción de Helck $(1983,97)$ propone leer la columna $N s ̌ j$. Habachi $(1972,44$, e) había considerado que el término wsr ("fuerte", Wb. I, 360) vinculaba al funcionario con actividades militares. Sin embargo, H. Fischer (1991, 29), basándose en evidencias contenidas en documentos de la dinas-

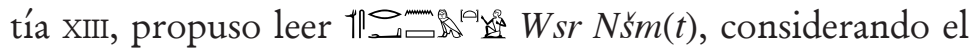
término wsr como parte del nombre y sosteniendo que la forma t保 $N s ̌ j($ Neshi) que aparece en la columna - y no atestiguada en otros documentos- sería un diminutivo.

Los ornamentos y las ropas con los que está representado el funcionario apuntan a su alto estatus, al igual que los títulos que posee (véase más abajo). En la imagen aparece de pie, orientado en dirección al texto, sosteniendo un abanico ceremonial en la mano izquierda que queda ubicado entre la columna donde

534) propusieron una traducción parecida: "Haré cada tarea para que me alaben ante el rey" ("Ich werde jeden Auftrag [so] erledigen, dass man mich vor dem König lobt”), y B. Hofmann (2004, 120): “Actuaré de acuerdo con todo lo que se me ha ordenado para ser alabado ante el rey" ("Ich werde entsprechend all dem, was mir befohlen wurde, handeln, so daß ich gelobt werde vor dem König”). Por su parte, P. Vernus $(1989,154)$ propuso, de modo muy cauteloso, restituir jr...t..nb... śqmq ir $R\left[{ }^{\complement} w \underline{d}\right] t[. k] n b[t]$, "iQue Ra haga todo lo que tú pidas!” "Que Rê réalise tout ce que tu ordonnes!”), pero reconociendo que no le satisfacía el modo de vincular esta restitución con la construcción siguiente: $h s t n t h r n s w$. M. Brose (TLA, 2014) propuso leer: $j r r=[j][h f t][w \underline{d}] . t[n ?]=[j ?]$ $n b[. t][r][h s w, t] n(, j) . t h r-n s w t$, “Actuaré de acuerdo con todo lo que me habían ordenado, a favor del rey” ("Ich werde handeln gemäß allem, was mir befohlen worden war, zur Gunst von Seiten des Königs”). Recientemente, B. Mathieu (2019, 713, n. 59) presentó la siguiente reconstrucción, que es la que sigo aquí: $j r=j[h f t w d] w . t[\sim n n=j]$ $n b[=j w 3 h] h s(w) . t(=j) h r n(y) . t-s w . t$, "Voy a actuar [de acuerdo con lo que me ha orden] ado [mi] señor, para que perdure mi favor junto al rey" ("Je vais agir [conformément à ce que m'a ordon]né [mon] seigneur pour que dure ma faveur d'auprès du roi”). J. M. Serrano Delgado $(2021,161)$ no incluye el colofón en su traducción. 
figuran su título y su nombre, y las líneas de texto. Posee una peluca, un gran collar sobre el pecho y una falda que, partiendo desde algo arriba de la cintura, alcanza las pantorrillas ("High-waisted kilt"; cf. Hallmann 2017, 2), probablemente asegurada con un cinto sash. El vientre prominente también es un rasgo que muestra su alto posicionamiento jerárquico. La figura sigue las líneas rígidas habituales evidenciadas en las representaciones del Reino Medio y el Segundo Periodo Intermedio, y que tendieron a hacerse más curvas y amplias durante el Reino Nuevo.

Las líneas 36-37 del texto brindan información sobre la posición del funcionario en la estructura administrativa y gubernamental de la Tebas de la época. Poseía varios títulos, algunos de los cuales presentan ciertas dificultades para un entendimiento completo. ${ }^{2}$ Los dos primeros están ampliamente documentados, son títulos de rango y solían poseerlos los funcionarios más importantes de la administración:

a) $\frac{-\pi}{\circ}(j) r(y)-p^{c} t$, "miembro de la élite" (Wb. II, 415.15-416.6; Jones 2000, núm. 1157; TLA lema núm. 94060).

b) $h 3 t j$-c , "líder de acción" (Wb. III, 25.7-26.2; Jones 2000, núm. 1858; TLA lema núm. 100520). Aquí sigo a W. Grajetzki $(2009,5)$ al traducir de este modo ("foremost of action"), ya que es un título de rango; si fuera un título de función, aparecería antepuesto al nombre de la persona, y en ese caso convendría traducirlo como "administrador local".

En las secuencias habituales del Reino Medio, los cuatro títulos de rango que poseían los funcionarios más encumbrados eran, precisamente, los ya mencionados iry- $p^{c} t$ y $h 3$ ty- ${ }^{c}$, y luego seguían, en orden: $s m r-w^{c} t y$, "amigo único (del rey)" y htmtbjty, "portador del sello del rey (gobernante)". Sin embargo,

${ }^{2}$ Sobre la evolución de los títulos de la administración durante el Segundo Periodo Intermedio y las dificultades que presentan, cf. Grajetzki 2010. 
en la secuencia aparecen los siguientes títulos a continuación de $h 3 t y-{ }^{-}$:

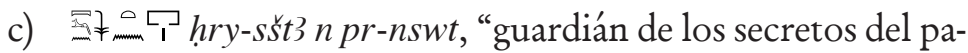
lacio" o "aquel que posee autoridad sobre los secretos del palacio” (TLA, lema núm. 400971; Jones 2000, núm. 2274). El título de hry-sšt 3 está documentado desde la dinastía III, y el término sśt t’ tiene que ver con la "protección” que "exige una distinción y una competencia particulares para ser revelada", es decir, se vincula con un conocimiento iniciático protegido que es común a las esferas religiosa y profesional (Loprieno 2001, 16). El término sšt 3 en este caso está escrito con el signo E15 冶 (EG, 459, E 15) -el perro (o chacal) recostado-, que remite a una escritura críptica del mismo. Suele estar acompañado de un genitivo descriptivo, como en este caso (Wb. IV, 298-299; FCD, 248), y se traduce como "secreto". El término hrry, — (Wb. III, 193; FCD, 174) posee el significado de "quien está por sobre”, "el que tiene autoridad sobre", que puede ser asimilado a "guardián”. Así, hrry-šst 3 podría ser traducido como "guardián de los secretos" o "aquel que tiene autoridad sobre los secretos”, en este caso "del palacio", con lo cual, posiblemente, remita a un vínculo estrecho entre el rey y este funcionario de alto rango.

d) que podría expresar alguna forma de autoridad territorial de un funcionario muy cercano al rey (Moreno García 2013, 144, n. 203; TLA lema núm. 851279; Ward 1982, núm. 1084).

Habachi (1972, 44, a) considera las dos expresiones precedentes como epítetos que solían ser atribuidos a los visires, aunque este último cargo no se evidencia durante el Segundo Periodo Intermedio. Sin embargo, pueden dar una idea del alto rango de Neshi y su cercanía estrecha con el rey. 


\section{A continuación, aparece:}

e) 乡 §ิ htmt-bjty, "portador del sello del rey (gobernante)". Para S. Quirke (1986, 123), el título no tiene carácter de rango sino de función, al menos durante el Reino Medio (cf. TLA lema núm. 400193; Wb. v, 638.12-14; Fischer 1996, 50; Jones 2000, núm. 2275). La escritura con el signo S3 \& es útil para su datación en el Segundo Periodo Intermedio (Grajetzki 2009, 12), cuando este título también comienza a ser adoptado por una mayor cantidad de individuos, lo cual es indicativo de los cambios que se observan en la administración en todos sus niveles (Grajetzki 2010, 309).

f) $\quad$ 에 $s b 3 t 3 w y h 3(w) t y$, “primer conductor/líder [?] de las Dos Tierras" (TLA lema núm. 400972, trad. M. Brose “dem ersten Lehrer / Lenker [?] der Beiden Länder”). ${ }^{3}$

${ }^{3}$ Éste es el título que más dificultades presenta, tanto por la falta de otras evidencias como por la amplia variedad de significados que posee el signo N14 , que puede leerse como $d w 3$ (Wb. v, 426), pero también como $s b 3$ (Wb. Iv, 82-83). Estos problemas obviamente se visualizan en las diversas traducciones de los académicos. Así, Habachi (1972, 44, n. b) leyó sb3 t’3wy ḩßwty y optó por "el conductor de las Dos Tierras y el líder" ("the conductor of the Two Lands and the leader"); H. Smith y A. Smith $(1976,62)$ lo tradujeron como "el alumno de las Dos Tierras" ("the pupil of the Two Lands"), considerando $s b 3$ como "alumno" (FCD, 219), posiblemente en referencia a los títulos datados en el Reino Medio con este significado, como $s b 3(w)$ $H r n b$ ' h, "alumno de Horus, señor del palacio", o bien sb3(w) Hr hnty pt, var. sb3w Hr $m$ hnty pt, "alumno de Horus que preside el cielo" (Fischer 1985, 32-33, núms. 1283a y 1283 b; también puede ser traducido como "instructor", como en $s b 3$ n $\mathrm{pr}$ - ' $n h$ h, "instructor de la Casa de Vida”; cf. Ward 1982, núm. 1282); U. Kaplony-Heckel y E. Kausen $(1985,533)$ tradujeron "el primer conductor/timonel de los Dos Países" (“dem ersten Steuermann der beiden Länder”), y Hofmann (2004, 120), "el jefe de los Dos Países” (“dem Leiter der beiden Länder”). Por su parte, D. Redford (1997, 15) lo tradujo tomando en cuenta el título precedente htmt(j)-bjtj, y los ligó de la siguiente manera: "el portador del sello del rey del Bajo Egipto de [?] 'Estrella-de-las-Dos-Tierras"” ("the seal-bearer of the King of Lower Egypt of [?] 'Star-of-the-Two-Lands”), basándose en que el título de "portador del sello" estaba atestiguado para funcionarios vinculados con barcos y expediciones, e interpretando 'Estrella-de-las-Dos-Tierras' como el nombre de una barca (30-31, n. 159). Sin embargo, esta propuesta no suele ser la adoptada por la mayor parte de quienes también tradujeron el texto. Considero relevante destacar que la expresión se encuentra inscrita en una estatua dedicada a Seth, el señor de Avaris, por el gobernante Nehesy (dinastía XIV) y que luego fuera usurpada por Merenptah 
g) rey]” (TLA lema núm. 400973; Jones 2000, núm. 848).

h) $\varlimsup_{\infty}$ jmy-r htmt, "supervisor de las cosas selladas" (TLA lema núm. 400044; Wb. v, 639.2; Ward 1982, núm. 367; Franke 1984, 113-114). Éste es el título de función de Neshi. Como ya se señaló, con éste también se le identifica en la inscripción ubicada del lado derecho de la imagen tallada en la estela. Prefiero seguir las razones planteadas por W. Grajetzki (2013, 238, n. 132) para optar por la traducción "supervisor de las cosas selladas" sobre la habitual de "tesorero jefe", con el fin de evitar posibles confusiones con la palabra "tesoro" ( $p r h \underline{h} \underline{d})$. Sobre el título, Vernus señaló su operatividad en diferentes niveles de la administración - a nivel tanto local, como regional y central-, con lo cual, para determinar la posición del sujeto dentro de la estructura administrativa, se vuelve pertinente ponderar los demás títulos que posee. En el caso de Neshi, es indudable que este individuo ejerció una de las más altas posiciones en la administración de Tebas y su área de influencia durante el reinado de Kamose (Vernus 1994, 259; Grajetzki 2009, 46; Shirley 2013, 552), prueba de lo cual es que este último le haya confiado la realización de la estela, y que fuera autorizado a tallar en ella su propia imagen. ${ }^{4}$ De este modo, aparece escrito en la línea 37; mientras que, en la columna junto al nombre, aparece en la variante ?

(Borchardt 1925, 88, núm. 538; Helck 1983, 48, núm. 66). En la parte delantera, del lado izquierdo, puede leerse: $n t r r$ $n f r s b 3 t 3 w y$ [z3?] $R^{\circ}$, "el buen dios, ¿conductor? ¿líder? de las Dos Tierras, ‘hijo de? Ra” (Borchardt 1925, 88, núm. 538). El problema aquí para datar esta inscripción subyace en la superposición de escrituras más antiguas con otras más recientes. Aquí pareciera constituir un epíteto. En cuanto al término $h 3(w)$ ty (Wb. III, 28), puede traducirse como "primero" o "el primer ..." en tanto adjetivo nisbe. Habachi (1972, 44, b) lo considera "un epíteto descriptivo de hombres vinculados al ejército" y califica a Neshi como un posible "funcionario de enlace" ("office de liaison"). También se traduce el título completo como "líder maestro de las Dos Tierras" (cf. Persons and Names of the Middle Kingdom 2021).

${ }^{4}$ Por cierto, no abundan las representaciones de funcionarios del Segundo Periodo Intermedio; sobre estas cuestiones, cf. Grajetzki 2009, 4. 
En síntesis, se puede afirmar que Neshi era uno de los dignatarios de mayor confianza de Kamose -y quizás el de más alto rango, equiparable a un visir- y que, en ejercicio de esa función, le fue ordenado elaborar la estela que sería exhibida en el templo de Karnak. No se puede descartar que también ocupara un alto cargo militar.

Ahora bien, ciertas evidencias, si bien escasas, brindan pistas sobre el papel de los funcionarios de alto rango de la Tebas del Segundo Periodo Intermedio. De hecho, se posee información de un "supervisor de las cosas selladas" llamado Teti que sirvió bajo el reinado de Neb-jeper-Ra Antef de la dinastía XVII y ostentaba los cuatro títulos de rango antes mencionados: "miembro de la élite", "líder de acción", "portador del sello del rey (gobernante)" y "amigo único [del rey]" (Grajetzki 2009, 65-66). Su tumba, localizada en Dra Abu el-Naga, se encuentra en las proximidades de la del rey, lo que demuestra el estrecho vínculo que existía entre ambos.

Ya R. Enmarch (2013) hizo referencia a los vínculos literarios entre las narrativas de las estelas de Kamose y otras más tempranas - del género de las Königsnovellen-y se enfocó en particular en los temas relacionados con las actividades militares y el tratamiento de los enemigos, aludiendo a que algunas de estas narraciones solían incluir alguna noticia sobre la ejecución de las estelas (Enmarch 2013, 258, n. 29). Ahora bien, aunque escasa, cierta evidencia muestra que los gobernantes de la Tebas del Segundo Periodo Intermedio, predecesores de Kamose, no eran ajenos a esta práctica: solían encargar estelas conmemorativas destinadas al templo de Karnak a funcionarios de alto rango, y tal circunstancia era registrada de modo explícito en los monumentos. P. Vernus analizó los fragmentos de dos estelas halladas en dicho templo. La realización de una de ellas, perteneciente al rey Sanj-en-Ra Mentuhotepi (dinastía XVI; PM II, 73; Luxor CL $223 \mathrm{G}$ ), le fue ordenada a un alto funcionario local de nombre Montu-user y, tal como explica Vernus (1989, 152-153), contiene la fórmula codificada atestiguada también - aunque con algunas variantes- en K2. De la otra estela sólo se conserva 
un fragmento de la sección inferior, y no se conoce ni su datación ni el nombre del rey que la ordenó, pero también muestra parcialmente una fórmula semejante a las antedichas. Asimismo, es interesante observar que en uno de los fragmentos (E) recuperados de la Tercera Estela de Kamose - también hallada en Karnak - y que lamentablemente se encuentra en muy mal estado de conservación, se preserva parte de la misma secuencia de títulos que posee Neshi, incluso en el mismo orden: (w+28) 兵 primer ...[?], supervisor de los amigos [del rey]. Yo dí [?]” (Gabolde 2005, 40; Van Siclen 2010, 356), lo que sería un probable indicio de una fórmula semejante en esta última estela. De todos modos, lo que hace única a $\mathrm{K} 2 \mathrm{es,} \mathrm{precisamente,} \mathrm{la} \mathrm{representa-}$ ción del funcionario en ese contexto.

\section{Hacia una explicación alternativa de la imagen de Neshi en K2}

Como ya señalé, las líneas 31-38 de K2 enmarcan la figura de Neshi y contienen las acciones que Kamose llevó a cabo en relación con el dios tutelar de Tebas a su regreso a la ciudad. Repasémoslas aquí: la primera refiere un acto ritual efectuado en el ámbito más sagrado del templo. El rey, quien es llamado "hijo de Amón", ofrece rituales al dios: quema incienso en su altar, y aquél le entrega el jepesh como reconocimiento a sus victorias. Se incluye la titulatura del rey, con su nombre de coronación - Wady-jeper-Ra- acompañado del epíteto "rey viviente" y su nombre de Hijo de Ra, Kamose [el fuerte], acompañado de calificativos redundantes acerca de sus cualidades positivas y como líder vencedor de los portadores del caos. La segunda acción incluye el decreto del rey por el cual se le ordena específicamente a Neshi realizar una estela donde se relaten las victorias del rey, y se le indica dónde debe ser colocada: en el templo de Karnak en Tebas, donde permanecerá "por los siglos de los siglos". Finalmente, se suma la respuesta 
de Neshi -ejecutada frente al rey-, que si bien presenta dificultades para una reconstrucción exacta, es muy factible que registre su aceptación de lo ordenado por el gobernante.

Un aspecto determinante en mi interpretación es la orientación de la figura y el abanico que el funcionario sostiene en la mano izquierda, que se encuentra directamente frente a la titulatura del rey. Como se ha sostenido, desde tiempos tempranos el nombre del rey solía estar ligado a otros símbolos que lo complejizaban y dimensionaban. En K2, el rey no está representado antropomórficamente, sino a través de dos de los nombres que componen su titulatura: el prenomen o nombre de coronación (Wady-jeper-Ra) y el nomen o nombre de nacimiento (Ka-mes[w], "el toro ha nacido", Kamose). En una discusión acerca de la naturaleza divina del rey -que excede el marco de este trabajo-, P. Frandsen $(2008,58)$ menciona, siguiendo a S. Morenz, los componentes de la persona del Amtsperson König: el $k$, el $b$ 3, el $3 h$, el $h 3 t$, la sombra y el nombre. De modo que, si el nombre resulta ser una propiedad inalienable e intrínseca del individuo-rey en su función específica, podemos interpretar que está consustanciado con el rey al igual que podría estarlo su representación antropomórfica, de modo semejante a como la palabra $p r$-3, que significa "la gran casa" - es decir, el palacio, y que derivara en la denominación "faraón"-, muestra la identificación entre rey y palacio y conforma una misma entidad consustanciada (Cervelló Autuori 1996, 201, n. 89).

Como ya señalé, el abanico ceremonial que Neshi sostiene en la mano está a la altura de la titulatura como símbolo que, además de su propia significación, vincula e integra la representación física de Neshi con la representación nominativa del rey.

Como indica E. Teeter (2010, 3, citando a Bell 1985), los abanicos ceremoniales de plumas, como el S35 $\uparrow$, var. S36 $\uparrow^{\text {( }}(w t$ "parasol de plumas de avestruz", término que asimismo significa "sombra”, EG 508), acompañaban la presencia de divinidades o de sus intermediarios en procesiones y festivales. También solían añadirse a la iconografía del rey desde tiempos muy 
tempranos como estandartes (sryt): ya las cabezas de maza de Escorpión y Narmer poseen representaciones de estos objetos ligados a actividades militares terrestres o navales (cf. también Faulkner 1941, 12-18). El término $\underline{t} 3 y$-sryt se traduce como “portador del estandarte” (FCD, 235; LED II, 225).

Ahora bien, otro tipo de abanico ceremonial era el hww, término que se traduce como "abanico", asociado a los conceptos de "proteger, salvaguardar" (S37 + , † "abanico de mango corto", como determinativo \-TLA lema núm. 114930; Wb. III, 246.9-10; FCD, 186; EG, 508; LED I, 354). Se compone de un mango corto en forma de flor de loto sobre el que se erige una única pluma. Durante el Reino Nuevo, a partir del reinado de Hatshepsut, los funcionarios de alto rango comenzaron a ser representados sosteniendo frente al rey este tipo de abanico, cuando también el título is is t’3y hw hr wnmy n nsw, "portador (lit.: 'aquel que porta') del abanico a la derecha del rey” comenzó a ser registrado. Una forma más temprana del título, $t 3 y$ hw, "portador del abanico" (TLA lema núm. 174410; Wb. III, 246.10; v, 348.4; LED II, 225), se evidencia durante el periodo precedente al reinado de Tutmosis II (Pomorska 1987, 32-35; Hartwig 2010, 163-164).

Ahora bien, el abanico ceremonial que está representado en $\mathrm{K} 2$ es singular: si bien está constituido por un mango corto que termina en forma de flor de loto, al igual que el hw, sobre él se erige lo que probablemente sean tres plumas (imagen 4). Como ya señalé, la rigidez en las formas es la habitual en las representaciones del Reino Medio. Además, debemos tener en cuenta el estrecho espacio entre el texto principal y la columna donde aparecen el nombre y título de Neshi, en medio de los cuales se sitúa el objeto que describo.

Por cierto, si se tomaran en cuenta el contexto de la representación de Neshi y el estrecho vínculo entre el rey y el funcionario - como lo muestran los títulos que ostenta-, ¿sería factible evaluar una explicación alternativa a las ya brindadas? Considero que sí. Recordemos que el texto señala que Neshi recibió una orden del rey y respondió afirmativamente, e 
indica explícitamente que lo hizo "delante de su majestad" (1. 38: “... hft hmm =f...”). La cumplimentación de la orden fue, precisamente, la confección de la estela y su ubicación en el templo de Karnak.

Si bien se sabe que la práctica de encomendar la realización de estelas a altos funcionarios de la corte tebana se daba ya durante el Segundo Periodo Intermedio, carecemos de representaciones contemporáneas de funcionarios en relación con el rey en contextos semejantes al de K2, con lo cual hay que recurrir a representaciones posteriores -datadas en el Reino Nuevo- y provenientes de otro contexto - tumbas y no templos- para sostener una nueva argumentación. De hecho, en las tumbas de funcionarios de alto rango del Reino Nuevo localizadas en Sheikh Abd el-Qurna, se registran representaciones de estos individuos portando un abanico hw delante del rey. En la TT 78 - perteneciente a Horemheb, un alto funcionario que sirvió en los reinados de Amenofis II, Tutmosis IV y Amenofis III, y que, entre otros títulos de rango, llevaba el ya mencionado de "portador del abanico a la derecha del rey” (Brack y Brack 1980) - se visualiza una escena similar a la de K2, a pesar de los daños que presentan las imágenes

\author{
IMAGEN 4. Abanico \\ ceremonial sostenido \\ por Neshi, detalle
}

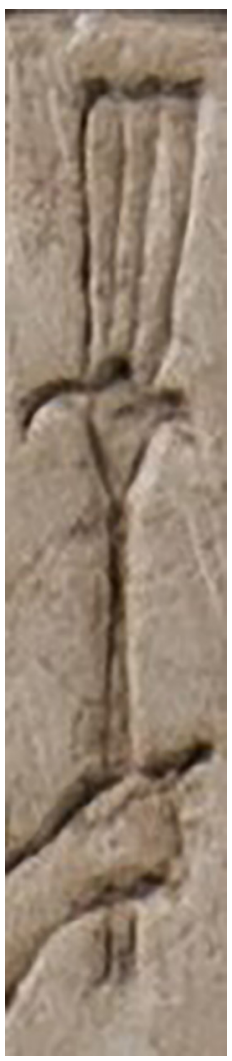

Fuente: Biston-Moulin y Thiers 2017, 62/J. Maucor. 
preservadas. En la representación ubicada en la sala transversal de la tumba, en la pared oeste del ala sur, el funcionario se encuentra de pie frente al rey (Tutmosis IV), mientras otros dos individuos sostienen sendos abanicos $h w$ en dirección a la figura regia (Brack y Brack 1980, lám. 86). Otra representación de índole semejante aparece en la inacabada TT 116, la cual se encuentra en mal estado de conservación, y de cuyo propietario no se conoce el nombre, pero sí información relativa a su servicio durante los reinados de Amenofis II y Tutmosis IV. En ella, el funcionario está de pie frente al rey, quien permanece sentado debajo de un baldaquino, con un abanico $h w$ en la mano (Hartwig 2010, 162 y figs. 5 y 6). Otras dos representaciones del rey y el funcionario interactuando se registran en la tumba del virrey de Kush Amenhotep, también llamado Huy (TT40), datada en el reinado de Tutanjamón (Davies y Gardiner 1926). Huy poseía, al igual que Horemheb, el título de "portador del abanico a la derecha del rey". En ambas representaciones se ve al funcionario en ocasión de ser recibido por el rey, que, al igual que en las imágenes anteriores, está sentado debajo de un baldaquino (imagen 5). El funcionario extiende hacia la figura regia el abanico ceremonial $h w$ con la mano derecha, mientras en la izquierda sostiene un cetro heqa, que denota su función como "virrey" de Kush. En el contexto general de orden funerario de la representación de la TT40, la presentación de Huy ante el rey tiene que ver directamente con la entrega de los productos de Asia y Nubia que hacían a sus funciones. En una de ellas, se preservan líneas de texto que mencionan alabanzas al rey en ocasión de la presentación del tributo asiático (Davies y Gardiner 1926, 29).

De este modo, las representaciones de estos funcionarios delante del rey pueden ser útiles para proponer una interpretación plausible acerca del sentido general de la imagen de Neshi en K2: en ellas intervienen el funcionario y el rey, el objeto que los vincula es un abanico ceremonial, y remiten a una acción encomendada por el rey o requerida por el cargo que desempeñan. 
IMAGEN 5. Representación de la TT40 donde se visualiza al funcionario frente al rey, detalle

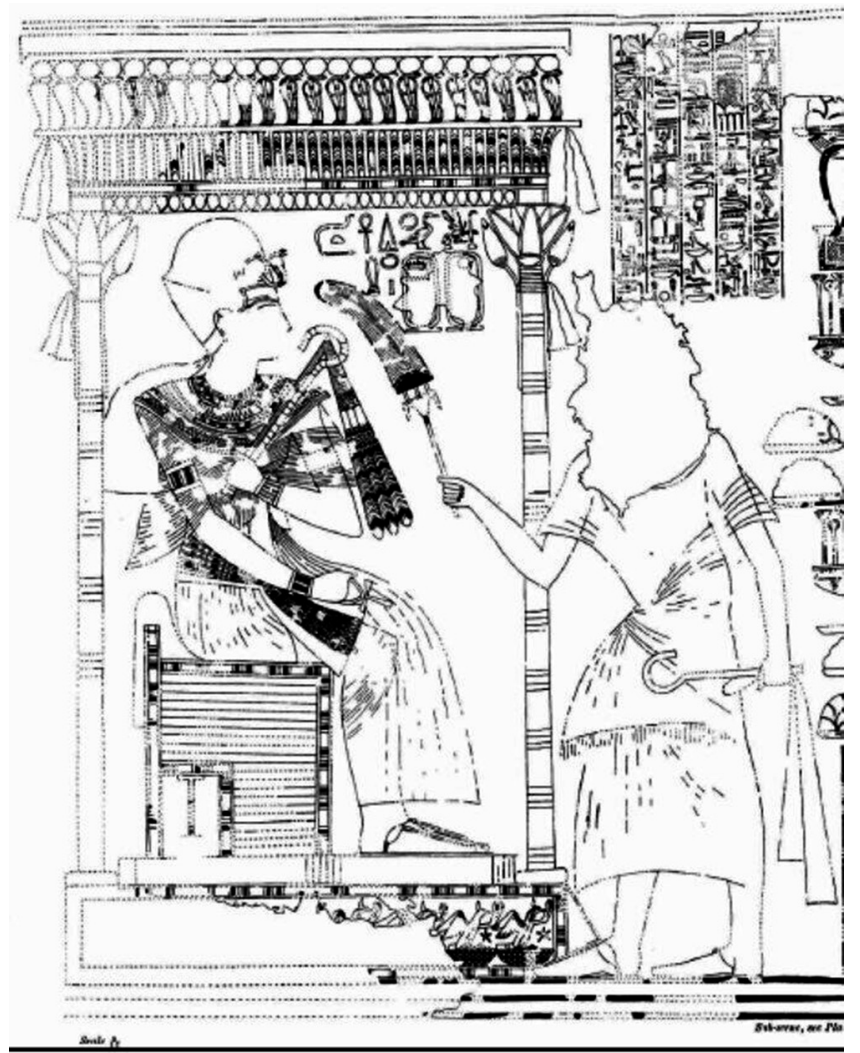

Fuente: Davies y Gardiner 1926, lám. xx. Cortesía de la Egypt Exploration Society.

Dicho esto, el contexto específico de K2 brinda la posibilidad de dar una explicación alternativa a la representación del funcionario que no se contradice con la posibilidad de que éste haya tenido la suficiente aquiescencia del rey como para ser incluido en la estela. Sin embargo, al tomar en cuenta lo ya mencionado (las evidencias que prueban la práctica 
de encomendar a funcionarios de alto rango la ejecución de estelas conmemorativas destinadas al templo de Karnak por los gobernantes de la Tebas del Segundo Periodo Intermedio, lo que permite suponer gran cercanía entre los "supervisores de las cosas selladas" y esos gobernantes, y que a posteriori se registren escenas donde el rey y sus más altos funcionarios interactúan y que incluyen un abanico ceremonial), es posible argumentar que la imagen de Neshi en la estela forma parte de una escena más amplia. De este modo, remite a un acto en el que intervienen el funcionario y el rey (aquí representado en forma nominativa), y es precisamente el texto el que da las pistas para su comprensión, ya que plasma el momento en que la orden de realizar la estela que se erigirá en Karnak es emitida por el rey y aceptada por Neshi, quien la recibe frente al rey -tal como dice el texto- portando un abanico ceremonial en la mano. Si bien mi explicación no deja de ser hipotética, se puede aventurar que quizá se trate de una de las representaciones más tempranas de este tipo de actos entre un funcionario del más alto rango y el rey, y a pesar de no disponer de paralelos contemporáneos con los cuales comparar la escena, es posible que interactúe directamente con la narrativa y con la estela al ser parte sustancial del monumento.

\section{Agradecimientos}

Este trabajo fue realizado durante la cuarentena impuesta por la pandemia de la covid-19, que impidió el acceso a bibliotecas físicas por largo tiempo. Por este motivo, quiero agradecer a quienes, a la distancia, me hicieron llegar material bibliográfico sustancial a través de una maravillosa red virtual de investigadores dedicados al Cercano Oriente Antiguo que comenzó a operar a través de Facebook cuando la cuarentena se impuso en el mundo. De este modo, ANE Researcher Quarantine "Library", tal el nombre de la red, configuró un sistema de interacción y colaboración académica nunca antes visto, con 
más de 1750 miembros a la fecha del envío de este trabajo (abril de 2020). No tengo más que palabras de gratitud para Nigel Strudwick, Ryan Thomas, Dmitry Sychev y Günther Livingstone-Thomas por facilitarme material indispensable para completar este trabajo. Además, va mi reconocimiento a John Baines por los comentarios acerca de la representación de Neshi que compartió desinteresadamente conmigo, así como también a los evaluadores anónimos provistos por la revista Estudios de Asia y África. Por cierto, los errores que puedan haberse deslizado son de mi absoluta responsabilidad.

\section{Obras de referencia y sus abreviaturas}

EG Gardiner, Alan H. 2001. Egyptian Grammar: Being an Introduction to the Study of Hieroglyphs. $3^{a}$ ed. revisada. Oxford: Griffith Institute, Ashmolean Museum.

FCD Faulkner, Raymond O. 1991. A Concise Dictionary of Middle Egyptian. $7^{\text {a }}$ ed. Oxford: Griffith Institute, Ashmolean Museum.

LED I Lesko, Leonard S. y Barbara S. Lesko. 2002. A Dictionary of Late Egyptian. Vol. I, $2^{\text {a }}$ ed. Providence, R. I.: B. C. Scribe Publications.

LED II Lesko, Leonard S. y Barbara S. Lesko. 2004. A Dictionary of Late Egyptian. Vol. II, 2a ed. Providence, R. I.: B. C. Scribe Publications.

PM II Porter, Bertha y Rosalind Moss. 1972. Topographical Bibliography of Ancient Egyptian Hieroglyphic Texts, Reliefs, and Paintings: II. Theban Temples. $2^{\mathrm{a}}$ ed. Oxford: Clarendon Press.

TLA Thesaurus Linguae Aegyptiae ${ }^{\circ} \mathrm{BBAW}$ - Ancient Egyptian Dictionary Project. 2014. https://aaew.bbaw.de/tla/servlet/ S05?d $=\mathrm{d} 001 \& \mathrm{~h}=\mathrm{h} 001$

Wb. Erman, Adolf y Hermann Grapow. 1926-1971. Wörterbuch der ägyptischen Sprache, Bd. I-VII. Berlín: Akademie. 


\section{Referencias}

Allam, Schafik. 1989. "Some Remarks on the Trial of Mose". The Journal of Egyptian Archaeology 75: 103-112. https://doi. org $/ 10.2307 / 3821902$

BeLL, Lanny. 1985. "Aspects of the Cult of the Deified Tutankhamun”. En Mélanges Gamal Eddin Mokhtar. Vol. 1, Bibliothèque d'étude 97. Editado por Paule Posener-Kriéger, 31-59. El Cairo: Institut français d'archéologie orientale du Caire.

Biston-Moulin, Sébastien y Christophe Thiers. 2017. Le Centre franco-égyptien d'étude des temples de Karnak. Livre du cinquantenaire 1967-2017. Luxor: Cfeetk.

Borchardt, Ludwig. 1925. Statuen und Statuetten von Königen und Privatleuten im Museum von Kairo, Nr. 1-1294. Parte 2: Text und Tafeln zu Nr. 381-653. Catalogue Général des Antiquités Égyptiennes du Musée du Caire 77. Berlín: Reichsdruckerei. https:// doi.org/10.11588/diglit.34231

Brack, Annelies y Artur Brack. 1980. Das Grab des Harembeb: Theben Nr. 78. Archäologische Veröffentlichungen 35. Maguncia: Philipp von Zabern.

Cervelló Autuori, Josep. 1996. Egipto y África: origen de la civilización y la monarquía faraónicas en su contexto africano. Aula Orientalis Supplementa 13. Barcelona: AUSA.

Davies, Nina de Garis y Alan H. Gardiner. 1926. The Tomb of Huy: Viceroy of Nubia in the Reign of Tut'ankbamun. The Theban Tombs Series $4^{\text {th }}$ Memoir. Londres: The Egypt Exploration Society. http://hdl.handle.net/1959.14/1770

Enmarch, Roland. 2013. "Some Literary Aspects of the Kamose Inscriptions”. The Journal of Egyptian Archaeology 99 (1): 253-263. https://doi.org/10.1177/030751331309900112

FaulKner, Raymond O. 1941. "Egyptian Military Standards". The Journal of Egyptian Archaeology 27 (1): 12-18. https://doi. org/10.1177/030751334102700104

FIscher, Henry G. 1985. Egyptian Titles of the Middle Kingdom: A Supplement to Wm. Ward's Index. Nueva York: Metropolitan Museum of Art.

FISCHER, Henry G. 1991. "Marginalia”. Göttinger Miszellen 122: 21-30. http://www.digizeitschriften.de/dms/resolveppn/?PID = PPN $522563589 \_0122 \mid \log 11$ 
FISCHER, Henry G. 1996. Varia Nova: Egyptian Studies III. Nueva York: Metropolitan Museum of Art.

FrandSEN, Paul J. 2008. "Aspects of Kingship in Ancient Egypt". En Religion and Power: Divine Kingship in the Ancient World and Beyond. Oriental Institute Seminars 4. Editado por Nicole Brisch, 47-73. Chicago: Oriental Institute of the University of Chicago.

Franke, Detlef. 1984. "Probleme der Arbeit mit altägyptischen Titeln des Mittleren Reiches”. Göttinger Miszellen 83: 103-124. http:// www.digizeitschriften.de/dms/resolveppn/?PID = PPN52256 $3589 \_0083 \mid \log 27$

GabOLDE, Luc. 2005. "Une troisième stèle de Kamosis?”. Kyphi : Bulletin du cercle lyonnais d'Égyptologie Victor Loret 4: 35-42.

Gardiner, Alan H. 1905. The Inscription of Mes. A Contribution to the Study of Egyptian Judicial Procedure. Untersuchungen zur Geschichte und Altertumskunde Ägyptens IV, núm. 3. Leipzig: J. C. Hinrichs.

Goedicke, Hans. 1971-1972. Reseña de The Ancient Near East. Supplementary Texts and Pictures Relating to the Old Testament, editado por James B. Pritchard. Journal of the American Research Center in Egypt 9, 150-153. https://doi.org/10.2307/40001073

Goedicke, Hans. 1995. Studies about Kamose and Abmose. Baltimore, MD: Halgo.

Grajetzki, Wolfram. 2009. Court Officials of the Egyptian Middle Kingdom. Londres: Duckworth.

GrajeTZKI, Wolfram. 2010. "Notes on Administration in the Second Intermediate Period". En The Second Intermediate Period (Thirteenth-Seventeenth Dynasties): Current Research, Future Prospects. Orientalia Lovaniensia Analecta, 192. Editado por Marcel Marée, 305-312. Lovaina: Peeters.

GrajetzKi, Wolfram. 2013. "Setting a State Anew: The Central Administration from the End of the Old Kingdom to the End of the Middle Kingdom”. En Ancient Egyptian Administration. Handbook of Oriental Studies-Handbuch der Orientalistik. Section 1, Ancient Near East (HdO), 104. Editado por Juan Carlos Moreno García, 215-258. Leiden: Brill. https://doi. org/10.1163/9789004250086_009

Habachi, Labib. 1972. The Second Stela of Kamose and his Struggle Against the Hyksos Ruler and his Capital. Abhadlungen des Deuts- 
chen Archäologischen Instituts Kairo- Ägyptologie Reihe: Band 8. Glückstadt: J.J. Augustin.

Hallmann, Aleksandra. 2017. "Clothing (non-royal), Pharaonic

Egypt". En The Encyclopedia of Ancient History, editado por Roger S. Bagnall, Kai Brodersen, Craige B. Champion, Andrew Erskine y Sabine R. Huebner, 1-9. DeKalb, GA: John Wiley \& Sons. https://doi.org/10.1002/9781444338386.wbeah30017 Hartwig, Melinda. 2010. "The Tomb of a h3ty-', Theban Tomb 116". En Offerings to the Discerning Eye: An Egyptological Medley in Honor of Jack A. Josephson. Culture and History of the Ancient Near East Vol. 38. Editado por Sue D’Auria, 159-167. Leiden: Brill. https://doi.org/10.1163/ej.9789004178748.i-362.58

Helck, Wolfgang. 1983. Historisch-biographische Texte der 2. Zwischenzeit und Neue Texte der 18. Dynastie. Kleine ägyptische Texte 6. Wiesbaden: Harrassowitz.

Hofmann, Beate. 2004. Die Königsnovelle: "Strukturanalyse am Einzelwerk". Ägypten und Altes Testament 62. Wiesbaden: Harrassowitz.

Jones, Dilwyn. 2000. An Index of Ancient Egyptian Titles, Epithets and Phrases of the Old Kingdom. BAR International Series 866 (I). Oxford: Archaeopress.

KaplONY-HeCKel, Ursula y Ernst Kausen. 1985. "Ägyptische historische Texte”. En Historisch-chronologische Texte III, editado por Otto Kaiser, 525-619. Gütersloh: Gütersloher Verlaghaus. https://doi.org/10.14315/9783641217631-001

LOPRIENO, Antonio. 2001. La pensée et l'écriture : Pour une analyse sémiotique de la culture égyptienne. París: Cybele.

Mathieu, Bernard. 2019. “Attaquer ou ne pas attaquer? Le pharaon Kamosis au coeur d'un conflit idéologique (avec une nouvelle traduction de la 'Geste de Kamosis')". En En détail - Philologie und Archäologie im Diskurs: Festschrift für Hans-W. Fischer-Elfert, editado por Marc Brose, Peter Dils, Franzisca Naether, Lutz Popko y Dietrich Raue, 703-718. Berlín: De Gruyter.

Moreno García, Juan Carlos. 2013. “The Territorial Administration of the Kingdom in the $3^{\text {rd }}$ Millennium". En Ancient Egyptian Administration. Handbook of Oriental Studies-Handbuch der Orientalistik. Section 1, Ancient Near East (HdO), 104. Editado por Juan Carlos Moreno García, 85-151. Leiden: Brill. https:// doi.org/10.1163/9789004250086_005 
Persons and Names of the Middle Kingdom, Versión 1. 2021. Título 1024. https://pnm.uni-mainz.de/title/1024

POMORSKA, Irena. 1987. Les flabelliferes à la droite du roi en Égypte ancienne. Varsovia: Éditions scientifiques de Pologne.

Posener, Georges. 1964. "Néshi du procès de Mès". Revue d'Égyptologie 16: 213-214.

Quirke, Stephen. 1986. “The Regular Titles of the Late Middle Kingdom”. Revue d'Égyptologie 37: 107-130. https://doi.org/10.2143/ RE.37.0.2011656

REDFORD, Donald. 1997. "Textual Sources for the Hyksos Period". En The Hyksos: New Historical and Archaeological Perspectives, editado por Eliezer D. Oren, 1-44. Filadelfia, PA: University of Pennsylvania.

Serrano Delgado, José Manuel. 2021, Textos para la historia antigua de Egipto. $2^{a}$ ed. Madrid: Cátedra.

ShIRLEy, J. J. 2013. "Crisis and Restructuring of the State: From the Second Intermediate Period to the Advent of the Ramesses". En Ancient Egyptian Administration. Handbook of Oriental Studies-Handbuch der Orientalistik. Section 1, Ancient Near East (HdO), 104. Editado por Juan Carlos Moreno García, 521606. Leiden: Brill. https://doi.org/10.1163/9789004250086_014

SMITH, Harry S. y Alexandrina Smith. 1976. "A Reconsideration of the Kamose Texts”. Zeitschrift für ägyptische Sprache und Altertumskunde 103 (1): 48-73. https://doi.org/10.1524/zaes.1976.103. jg. 48

TEETER, Emily. 2010. "Feathers”. En UCLA Encyclopedia of Egyptology, editado por Willecke Wendrich. Los Ángeles. http://digital2. library.ucla.edu/viewItem.do?ark $=21198 /$ zz00256swd

VAn Siclen, Charles C. 2010. "The Third Stela of Kamose". En The Second Intermediate Period (Thirteenth-Seventeenth Dynasties): Current Research, Future Prospects. Orientalia Lovaniensia Analecta 192. Editado por Marcel Marée, 355-358. Lovaina: Peeters. Vernus, Pascal. 1989. "La stèle du pharaon Mntw-Htpi à Karnak : Un nouveau témoignage sur la situation politique et militaire au début de la D. P. I. (Planches 6-7)". Rerue d'Égyptologie 40: 145-161. https://doi.org/10.2143/RE.40.0.2011598

Vernus, Pascal. 1994. "Observations sur le titre imy-r3 htmt 'Directeur du trésor"”. En Grund und Boden in Altägypten (Rechtliche und sozio-ökonomische Verhältnisse), Akten des internationalen 
Symposions, Tübingen 18-20. Juni 1990. Untersuchungen zum Rechtsleben im alten Ägypten 2. Editado por Schafik Allam, 251-260. Tubinga: Selbstverlag des Herausgebers.

WARD, William A. 1982. Index of Egyptian Administrative and Religious Titles of the Middle Kingdom. Beirut: American University of Beirut.

Roxana Flammini es historiadora, especializada en egiptología. Estudió la carrera de historia en la Facultad de Filosofía y Letras de la Universidad de Buenos Aires (prof. 1988, diploma de honor; lic. 1994; doc. 2007). Su trabajo de investigación se focaliza en las relaciones entre el antiguo Estado egipcio y las ciudades levantinas y nubias, así como en enfoques teóricos (análisis de sistemas-mundo), y más recientemente en el estudio de las estelas monumentales regias de la transición entre el Segundo Periodo Intermedio y el Reino Nuevo. Actualmente dicta el curso de Historia del Antiguo Cercano Oriente en la Universidad Nacional de Luján y dirige el Instituto de Investigaciones de la Facultad de Ciencias Sociales de la Universidad Católica Argentina, donde además se desempeña como investigadora del Conicet. Fundó el Centro de Estudios de Historia del Antiguo Oriente (CEHAO) en la Universidad Católica Argentina en 2002 y la revista especializada Antiguo Oriente en 2003. Forma parte del comité editorial de las Ancient Near East Monograph Series/Monografías sobre el Antiguo Cercano Oriente editadas por la Society of Biblical Literature (SBL) y el CEHAO. Fue miembro de la Iniciativa de Cooperación Internacional de la SBL. Ha publicado libros, artículos en revistas y capítulos en libros sobre sus temas de investigación.

http://orcid.org/0000-0002-4839-3646 roxflamm@gmail.com 